\title{
Optimum Battery Weight for Maximizing Available Energy in UAV-Enabled Wireless Communications
}

\author{
Hua Yan, Student Member, IEEE, Shuang-Hua Yang, Yunfei Chen, and Suhaib A. Fahmy, Senior Member, IEEE
}

\begin{abstract}
Battery-powered unmanned aerial vehicles (UAVs) have been widely used as enablers of wireless networks. In this letter, the optimal battery weight for UAV-enabled wireless sensor networks is studied. The energy available for communication by considering propulsion energy consumption is maximized. Both numerical and approximate solutions to the optimal battery weight are derived. Numerical results show that both vertical and horizontal flight speeds and the gross weight of the UAV have great impact on the optimal battery weight.
\end{abstract}

Index Terms-Battery capacity, electric propulsion system (EPS), power consumption, unmanned aerial vehicle (UAV).

\section{INTRODUCTION}

B ATTERY-POWERED electric propulsion systems have been widely used for unmanned aerial vehicles (UAVs) [1]. One challenge for battery-powered UAV communications is the choice of battery weight, as a larger battery weight leads to higher battery capacity for longer flight [3] but also heavier UAV to consume more propulsion power. There may exist an optimal battery weight for the best UAV performance, and this applies to both single and multiple UAV applications [2].

Several works have been conducted on battery-powered UAV electric propulsion systems (EPS). For example, the authors in [4] proposed a systematic design for EPS considering the UAV's payload capacity, flight time and battery pack. In [5], several factors, such as the battery-dumping system and battery packs, were studied to analyse the performance of UAVs. The authors in [6] extended the endurance of battery-powered UAVs by grouping battery packs, and the logarithmic growth trend of durability extension was obtained. In [7], different factors in the design of the EPS for UAVs were investigated. In [8], empirical power consumption models for an Intel Aero Ready to Fly Drone were derived for energy efficient UAV mission planning. All these works have provided very useful insights on the design of battery-powered UAVs. However, none of these works has considered optimal battery weight by accounting for UAV propulsion power consumption, which affects the UAV flight and hence communications performance in practice.

In this work, we study the optimal battery weight in UAVenabled wireless sensor networks. Both vertical and horizontal flights are considered. Numerical results show that the best battery weight that optimizes the flight performance is determined

This research is supported by the National Natural Science Foundation of China under Grant (61873119 and 92067109), the National Key R\&D Program of China under Grant No. 2019YFC0810705 and the Science and Technology Innovation Commission of Shenzhen under Grant No. KQJSCX20180322151418232. (Corresponding author: Shuang-Hua Yang)

H. Yan and Y. Chen are with the School of Engineering, University of Warwick, Coventry, U.K. CV4 7AL (e-mail: Hua.Yan@warwick.ac.uk and Yunfei.Chen@warwick.ac.uk).

S. Yang is with the Department of Computer Science and Engineering, Southern University of Science and Technology, Shenzhen 518000, China. (email: yangsh@sustech.edu.cn)

S. A. Fahmy is with King Abdullah University of Science and Technology (KAUST), Thuwal, Saudi Arabia and the School of Engineering, University of Warwick, Coventry, U.K. (e-mail:suhaib.fahmy@kaust.edu.sa)

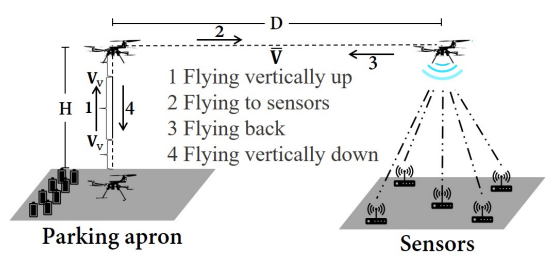

Fig. 1. System model.

by the flight height, flight distance, vertical/horizontal flight speed and the gross mass of the UAV excluding battery mass.

\section{SySTEM MODEL}

Consider the system shown in Fig. 1, where a set of batteries on the parking apron are used as energy for UAV communications. A battery-powered UAV is first equipped with the batteries through an automatic replacement mechanism [9], then flies vertically up to an altitude of $H$ followed by a horizontal flight of distance $D$ to the remote sensors with propulsion consumption, denoted as Stages 1 and 2 in Fig. 1. Upon arrival, the UAV consumes an energy of $E_{A 2 G}$ for different communications tasks, such as data collection/transmission and wireless power transfer (WPT) [10] - [11]. Finally, the UAV flies back to the parking apron to replace its batteries for the next flight, denoted as Stages 3 and 4 symmetric to Stages 2 and 1, respectively.

In this study, it is assumed that the mass of the UAV, including that of the fuselage and communications system but excluding the battery pack, is $m_{0}$, and the mass of the battery pack is $m_{b}$. Commonly used lithium polymer (LiPo) batteries are considered because of their high energy density [12]. Thus, the total mass of the UAV is $m=m_{0}+m_{b}$. Using results in [3], [7] and [13], the energy capacity $E_{b}$ in $W \cdot s$ as a function of the battery mass $m_{b}$ can be derived as

$$
E_{b}\left(m_{b}\right)=\rho_{e} * 3600 * m_{b} * \eta_{D C-D C}, 0 \leq m_{b} \leq m_{b, \max },
$$

where $\rho_{e}$ is the energy density $\left(W \cdot h r / k g\right.$ ) [3], $\eta_{D C-D C}$ is the DC-DC conversion efficiency ranging from 0.9 to 0.95 [14], $m_{b, \max }$ is the maximum battery mass [7] limited by the rotor thrust.

Remarks: Note that, from [3], the energy density of the LiPo batteries is currently $150 \mathrm{Wh} / \mathrm{kg}$, and can be increased to $250 \mathrm{Wh} / \mathrm{kg}$. We set it to $150 \mathrm{Wh} / \mathrm{kg}$ in this work. In the case when the parking apron has a height of $H$, the vertical flight can be ignored. The following results are still valid by ignoring Stages 1 and 4. Also, in the case when there is no automatic battery replacement mechanism [9], charging through a charging station can be considered.

For the manoeuvring of the UAV, the authors in [15] derived an analytical propulsion power consumption model for rotary- 
wing UAVs flying at a speed of $V$ with fixed height and rotor thrust as

$$
P(V)=P_{0}\left(1+\frac{3 V^{2}}{U_{t i p}^{2}}\right)+P_{1}\left(\sqrt{1+\frac{V^{4}}{4 v_{0}^{4}}}-\frac{V^{2}}{2 v_{0}^{2}}\right)^{\frac{1}{2}}+\frac{d_{0} \rho s A V^{3}}{2},
$$

where $P_{0}=\frac{\delta}{8} \rho s A \Omega^{3} R^{3}$ and $P_{1}=(1+k) \sqrt{\frac{(m g)^{3}}{2 \rho A}}$ are two constants related to the physical properties of the UAV and the flight environment, including profile drag coefficient $\delta$, air density $\rho$, rotor solidity $s$, rotor disc area $A$, blade angular velocity $\Omega$, rotor radius $R$, incremental correction factor to induced power $k$ and gravity acceleration $g, U_{t i p}$ denotes the tip speed of the rotor blade, $v_{0}=\sqrt{\frac{m g}{2 \rho A}}$ and $d_{0}$ are the mean rotor induced velocity and the fuselage drag ratio, respectively. Details can be found in [15].

For vertical flight, the authors in [16] derived the power consumption model as

$$
P_{v}\left(V_{v}, a\right)=P_{2}+\frac{T}{2}\left(V_{v}+\sqrt{V_{v}^{2}+\frac{2 T}{\rho A}}\right),
$$

where $T=m(a+g)$ is the rotor thrust, $V_{v}$ is the velocity of vertical flight, $P_{2}=\frac{\delta}{8} \rho s A \Omega^{3} R^{3}+k \sqrt{\frac{(m g)^{3}}{2 \rho A}}, g=9.8 \mathrm{~m} / \mathrm{s}^{2}$ is the gravitational acceleration, $a>0$ is the acceleration for ascending and $a<0$ for descending. We will calculate the propulsion energy consumption using these models.

In general, the performance of a UAV depends on the battery weight $m_{b}$. For small $m_{b}$, the total mass $m$ is small so that the amount of energy consumed by UAV manoeuvring operations is small. However, the available energy $E_{b}$ is also small, which results in shorter flight time or less energy for communications. There may exist an optimal $m_{b}$.

Denote the transmit power at the UAV as $P_{\text {uav }-t}$ in $\mathrm{dB}$. The received power at the ground sensor considering path loss [17] is

$$
P_{r}=P_{u a v-t}-\frac{A_{0}}{1+a_{0} \mathrm{e}^{-b_{0}\left(\theta_{0}-a_{0}\right)}}-B_{0},
$$

where $A_{0}=\eta_{L O S}-\eta_{N L O S}, B_{0}=20 \lg (H)+20 \lg (4 \pi f / c)+$ $\eta_{N L O S}, c$ is the speed of light, $\theta_{0}$ is the elevation angle, $\eta_{L O S}$, $\eta_{N L O S}, a_{0}$ and $b_{0}$ are constants related to the propagation environments. The achievable rate in bits/ $\mathrm{Hz}(\mathrm{bs} / \mathrm{Hz})$ is

$$
R=\tau \log _{2}\left(1+\frac{10^{\frac{P_{r}}{10}}}{10^{\frac{\sigma^{2}}{10}}}\right)
$$

where $\tau=\frac{E_{A 2 G}}{10^{\frac{P_{u a v-t}}{10}}+P(0)}$ is the time for data transmission while hovering, $P(0)$ is the hovering power when the speed is zero $\left(V=0\right.$ in (2)), and $\sigma^{2}$ is the received noise power at the sensor.

\section{Optimization OF BATtery Weight}

In this section, we will maximize $E_{A 2 G}$ to derive the optimal battery weight. $E_{A 2 G}$ is given by

$$
E_{A 2 G}=E_{b}\left(m_{b}\right)-2 \int_{0}^{T_{v}} P_{v}\left(V_{v}, a\right) d t-2 \int_{0}^{T_{h}} P(\bar{V}) d t,
$$

where 2 comes from the symmetric process of Stages 1 and 4, Stages 2 and 3, $T_{v}=\frac{H}{V_{v}}+\frac{V_{v}}{a}$ is the time for ascending in Stage 1 or the time for descending in Stage 4, assumed to be symmetric, $T_{h}=\frac{D}{\bar{V}}$ is the time for horizontal flight in Stage 2 or $3, \bar{V}$ is the mean velocity used to calculate the propulsion energy

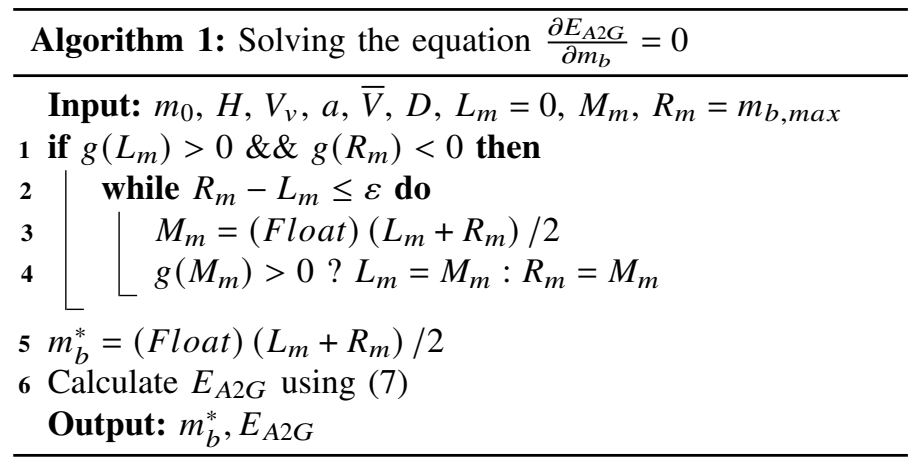

without considering the acceleration or deceleration, as they are relatively small compared with the long flight at constant speed. The calculation of $\int_{0}^{T_{v}} P_{v}\left(V_{v}, a\right) d t$ is divided into three parts, acceleration, constant speed and deceleration, in which the rotor thrust $T$ are $m(a+g), m g$ and $m(g-a)$, respectively. Thus, one has

$$
E_{A 2 G}=E_{b}\left(m_{b}\right)-E_{v F}-E_{h F},
$$

where

$$
\begin{aligned}
E_{v F}= & 2 P_{2}\left(\frac{H}{V_{v}}+\frac{V_{v}}{a}\right)+m g\left(\sqrt{V_{v}^{2}+\frac{2 m g}{\rho A}}\right)\left(\frac{H}{V_{v}}-\frac{V_{v}}{a}\right) \\
& +m(a+g)\left(\frac{V_{v}}{2 a} \zeta_{1}+\frac{m(a+g)}{a \rho A} \ln \frac{V_{v}+\zeta_{1}}{\xi_{1}}\right)+m g H \\
& +m(g-a)\left(\frac{V_{v}}{2 a} \zeta_{2}+\frac{m(g-a)}{a \rho A} \ln \frac{V_{v}+\zeta_{2}}{\xi_{2}}\right) \\
E_{h F} & =2\left[P_{0}\left(1+\frac{3 \bar{V}^{2}}{U_{\text {tip }}^{2}}\right)+P_{1} \varsigma_{1}+\frac{d_{0} \rho s A \bar{V}^{3}}{2}\right] \frac{D}{\bar{V}}
\end{aligned}
$$

are the total energy for vertical and horizontal flights, respectively, $\zeta_{1}=\sqrt{V_{v}^{2}+\frac{2 m(a+g)}{\rho A}}, \quad \xi_{1}=\sqrt{\frac{2 m(a+g)}{\rho A}}, \quad \zeta_{2}=$ $\sqrt{V_{v}{ }^{2}+\frac{2 m(g-a)}{\rho A}}, \xi_{2}=\sqrt{\frac{2 m(g-a)}{\rho A}}$ and $\varsigma_{1}=\sqrt{\sqrt{1+\frac{\bar{V}^{4}}{4 v_{0}^{4}}}-\frac{\bar{V}^{2}}{2 v_{0}^{2}}}$. To maximize $E_{A 2 G}$, one further has

$$
\frac{\partial E_{A 2 G}}{\partial m_{b}}=\rho_{e} * 3600 * \eta_{D C-D C}-\frac{\partial E_{v F}}{\partial m_{b}}-\frac{\partial E_{h F}}{\partial m_{b}},
$$

where

$$
\frac{\partial E_{h F}}{\partial m_{b}}=\frac{\sqrt{2} D}{\bar{V}}(1+k) g\left(\sqrt{\varsigma_{2}-\bar{V}^{2}}+\frac{m^{2} g^{2}}{2 \varsigma_{2} \rho^{2} A^{2} \sqrt{\varsigma_{2}-\bar{V}^{2}}}\right),
$$

$$
\begin{aligned}
& \frac{\partial E_{v F}}{\partial m_{b}}=g\left(\frac{H}{V_{v}}-\frac{V_{v}}{a}\right)\left(\sqrt{V_{v}^{2}+\frac{2 m g}{\rho A}}+\frac{m g}{\rho A \sqrt{V_{v}^{2}+\frac{2 m g}{\rho A}}}\right) \\
& +\frac{V_{v}}{2}\left(\zeta_{1}-\zeta_{2}\right)-\frac{V_{v} g}{2 a}\left(\zeta_{1}+\zeta_{2}\right)+\left(\frac{H}{V_{v}}+\frac{V_{v}}{a}\right) \frac{3 k \sqrt{m g^{3}}}{\sqrt{2 \rho A}} \\
& +\frac{m V_{v}}{2 a \rho A}\left(\frac{(a+g)^{2}}{\zeta_{1}}+\frac{(g-a)^{2}}{\zeta_{2}}\right)+g H \\
& +\frac{2 m}{a \rho A}\left((a+g)^{2} \ln \frac{V_{v}+\zeta_{1}}{\xi_{1}}+(g-a)^{2} \ln \frac{V_{v}+\zeta_{2}}{\xi_{2}}\right) \\
& +\frac{m^{2}}{a \rho^{2} A^{2}}\left(\frac{(a+g)^{3}}{\left(V_{v}+\zeta_{1}\right) \zeta_{1}}-\frac{(a+g)^{3}}{\xi_{1}^{2}}+\frac{(g-a)^{3}}{\left(V_{v}+\zeta_{2}\right) \zeta_{2}}-\frac{(g-a)^{3}}{\xi_{2}^{2}}\right)
\end{aligned}
$$




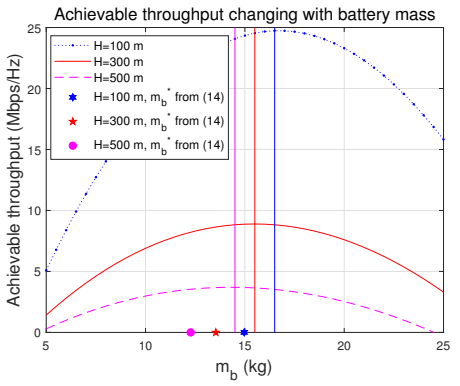

(a) Achievable throughput changing with $m_{b}$

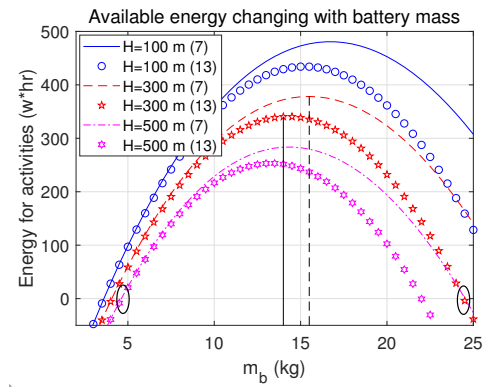

(b) $E_{A 2 G}$ changing with $m_{b}$

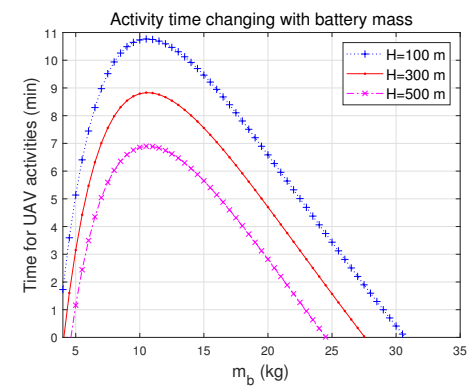

(c) Activity time $\tau$ changing with $m_{b}$

Fig. 2. Throughput, $E_{A 2 G}$ and $\tau$ changing with $m_{b}$.

$\varsigma_{2}=\sqrt{4 v_{0}{ }^{4}+\bar{V}^{4}}$. Taking the second-order derivative of (7), one has

$$
\frac{\partial^{2} E_{A 2 G}}{\partial m_{b}{ }^{2}}=-\left(\frac{\partial^{2} E_{v F}}{\partial m_{b}{ }^{2}}+\frac{\partial^{2} E_{h F}}{\partial m_{b}{ }^{2}}\right), 0 \leq m_{b} \leq m_{b, \max } .
$$

Using the energy density of $150 \mathrm{Wh} / \mathrm{kg}$ in [3] and the parameters from Table $\mathrm{I}$ in [15], it is found that $\frac{\partial^{2} E_{A 2 G}}{\partial m_{b}{ }^{2}}<0$, when $m_{b}$ is between 0 and $m_{b, \max }=20 \mathrm{~kg}$ [7]. This implies that the function $E_{A 2 G}$ has a unique maximum at $m_{b}=m_{b}^{*}$. It is challenging to obtain the exact solution $m_{b}^{*}$ by solving the equation $\frac{\partial E_{A 2 G}}{\partial m_{b}}=0$. Denote $g\left(m_{b}\right)=\frac{\partial E_{A 2 G}}{\partial m_{b}}$. This can be solved using the binary search in Algorithm 1 .

In Algorithm 1, $\varepsilon>0$ is the given precision tolerance. Thus, its complexity is $O\left(\log _{2} \frac{m_{b, \max }}{\varepsilon}\right)$. Note that, if $g\left(L_{m}=0\right)>0$ and $g\left(R_{m}\right) \geq 0, E_{A 2 G}$ will achieve its maximum at $m_{b}=R_{m}$. If $g\left(L_{m}=0\right) \leq 0$ and $g\left(R_{m}\right)<0$, it will achieve its maximum at $m_{b}=L_{m}$, but $m_{b}=L_{m}=0$. Once the maximum energy of $E_{A 2 G}$ is obtained, the maximum achievable throughput can be derived using (5).

Next, consider an approximation. In (2), $\left(\sqrt{1+\frac{V^{4}}{4 v_{0}^{4}}}-\frac{V^{2}}{2 v_{0}^{2}}\right)^{1 / 2}$ can be approximated as $\frac{v_{0}}{V}$ by applying the first-order Taylor approximation $(1+x)^{1 / 2} \approx 1+\frac{1}{2} x$ when $\left(\frac{v_{0}}{V}\right)^{4} \ll 1$. Then,

$$
E_{h F} \approx \frac{2 P_{0} D}{\bar{V}}\left(1+\frac{3 \bar{V}^{2}}{U_{t i p}}\right)+\frac{(1+k) m^{2} g^{2} D}{\rho A \bar{V}^{2}}+d_{0} \rho s A \bar{V}^{2} D .
$$

Also, if the acceleration time during vertical flight is less than that during constant speed, the consumption during vertical flight can be calculated using a mean velocity of $\overline{V_{v}}$ as

$$
E_{v F} \approx\left(2 P_{2}+m g \overline{V_{v}}+m g \sqrt{{\overline{V_{v}}}^{2}+\frac{2 m g}{\rho A}}\right) \frac{H}{\overline{V_{v}}} .
$$

Denote $E_{v F}$ as $h\left(m_{b}\right)$ and use the second-order Taylor approximation at $m_{b}=0$, one has

$$
E_{v F} \approx h(0)+h^{\prime}(0) m_{b}+\frac{1}{2} h^{\prime \prime}(0) m_{b}^{2}
$$

where $h(0)=\left(\frac{\delta}{4} \rho s A \Omega^{3} R^{3}+2 k \sqrt{\frac{\left(m_{0} g\right)^{3}}{2 \rho A}}+m_{0} g \overline{V_{v}}+m_{0} g \zeta_{3}\right) \frac{H}{V_{v}}$, $\zeta_{3}=\sqrt{{\overline{V_{v}}}^{2}+\frac{2 m_{0} g}{\rho A}}, h^{\prime}(0)=\frac{3 k g H}{2 \overline{V_{v}}} \sqrt{\frac{2 m_{0} g}{\rho A}}+g H+\frac{g H \zeta_{3}}{\overline{V_{v}}}+\frac{m_{0} g^{2} H}{\overline{V_{v}} \rho A \zeta_{3}}$ and $h^{\prime \prime}(0)=\frac{3 k g H}{4 \overline{V_{v}}} \sqrt{\frac{2 g}{m_{0} \rho A}}+\frac{2 g^{2} H}{\overline{V_{v}} \rho A \zeta_{3}}+\frac{m_{0} g^{3} H}{\overline{V_{v}} \rho^{2} A^{2} \zeta_{3}^{3}}$. Using (10) and (12), (7) can be rewritten as

$$
\begin{aligned}
E_{A 2 G} \approx & -\left(\frac{1}{2} h^{\prime \prime}(0)+\Phi\right) m_{b}^{2}-d_{0} \rho s A \bar{V}^{2} D \\
& +\left(\rho_{e} * 3600 * \eta_{D C-D C}-h^{\prime}(0)-2 \Phi m_{0}\right) m_{b} \\
& -\frac{2 P_{0} D}{\bar{V}}\left(1+\frac{3 \bar{V}^{2}}{U_{t i p}{ }^{2}}\right)-\Phi m_{0}^{2}-h(0),
\end{aligned}
$$

where $\Phi=\frac{(1+k) g^{2} D}{\rho A \bar{V}^{2}}$. From (13), the optimal weight is

$$
m_{b}^{*} \approx \frac{\rho_{e} * 3600 * \eta_{D C-D C}-h^{\prime}(0)-2 \Phi m_{0}}{h^{\prime \prime}(0)+2 \Phi} .
$$

\section{NumericAl Results AND Discussion}

In this section, numerical examples are given to show the optimal battery mass. In the examples, we set $\rho_{e}=150 \mathrm{Wh} / \mathrm{kg}$ [3], $\eta_{D C-D C}=0.9$ [14], $m_{0}=5.5 \mathrm{~kg}, m_{b, \max }=20 \mathrm{~kg}$ [7], $a=2 \mathrm{~m} / \mathrm{s}^{2}, P_{\text {uav-t }}=40 \mathrm{dBm}, f=2 \mathrm{GHz}, \sigma^{2}=-80 \mathrm{dBm}$, $D=40 \mathrm{~km}, V_{v}=4 \mathrm{~m} / \mathrm{s}$ and $\bar{V}=V=25 \mathrm{~m} / \mathrm{s}$. Also, a suburban environment is considered for communication, where $\eta_{L O S}=$ $0.1 \mathrm{~dB}, \eta_{N L O S}=21 \mathrm{~dB}, a_{0}=5.0188, b_{0}=0.3511$ [17], and other parameters of UAV are given in Table I of [15].

Fig. 2(a) shows the achievable throughput versus the battery mass when the flight height changes from $100 \mathrm{~m}$ to $500 \mathrm{~m}$. Consider one sensor below the UAV as an example, i.e., $\theta_{0}=0$. One sees that the achievable throughput increases first and then decreases when the battery mass increases. This is consistent with the analysis in Sections II and III. The higher the altitude $H$, the smaller the optimum $m_{b}$ and the achievable throughput will be. For example, when $H=500 \mathrm{~m}$, the optimal $m_{b}$ is about $14.5 \mathrm{~kg}$, which is smaller than $16.5 \mathrm{~kg}$ when $H=100 \mathrm{~m}$. This is because larger height consumes extra energy such that $E_{A 2 G}$ is reduced. For a fixed $H$, the optimal $m_{b}^{*}$ exists indicated by three straight lines. One can see that the values from approximation are smaller than those from Algorithm 1. This is because when $\overline{V_{v}}$ is set to $V_{v}$, the approximation in (12) is larger than the actual value. Meanwhile, the approximation in (10) is also larger than the actual value, thus reducing $E_{A 2 G}$.

Fig. 2(b) shows $E_{A 2 G}$ versus $m_{b}$. Similar observations can be made, because the throughput is proportional to $E_{A 2 G}$ in this case. In this figure, (7) and (13) are used to compare the numerical results of $E_{A 2 G}$ with the approximate results. Take $H=300 m$ as an example. The gap between the optimal $m_{b}$ using (7) and the one using (13) is about $1.5 \mathrm{~kg}$, almost consistent with the result in Fig. 2(a). Also, zero-crossing points 


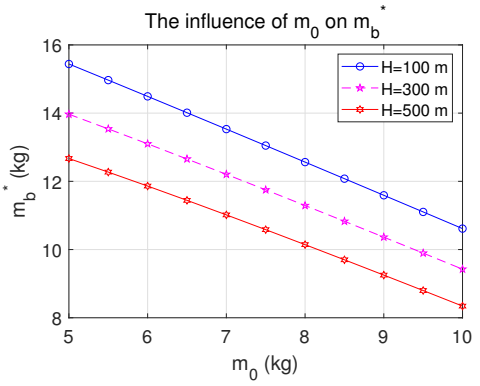

(a) $m_{b}^{*}$ changing with $m_{0}$

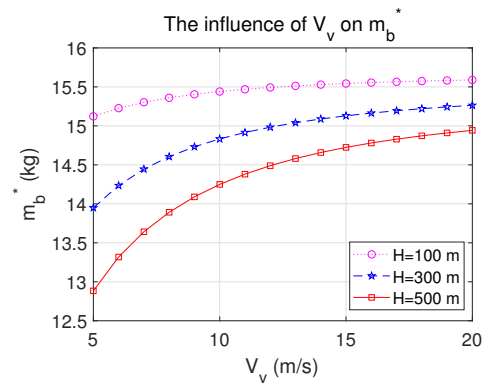

(b) $m_{b}^{*}$ changing with $V_{v}$

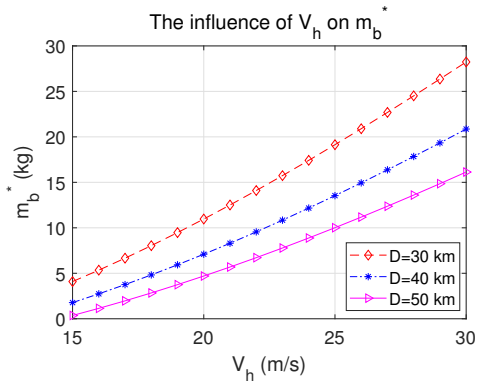

(c) $m_{b}^{*}$ changing with $V_{h}$

Fig. 3. The influence of $m_{0}, V_{v}$ and $V_{h}$ on $m_{b}^{*}$.

marked with ellipse in Fig. 2(b) indicate that $E_{A 2 G}=0$ so that the energy provided by the battery can only be used for manoeuvre, not for UAV communications. Note that, for a given task, where $E_{A 2 G}$ is certain and smaller than the peak value in Fig. 2(b), the optimal battery weight can be obtained by discussing the root of the equation (13) with $m_{b}^{*}$ as the unknown.

Fig. 2(c) shows the time for UAV activities using the same parameters as Fig. 2(a) and Fig. 2(b). One can see that, under the same conditions the optimal $m_{b}$ is about $10.5 \mathrm{~kg}$ now, smaller than that in Fig. 2(a) and Fig. 2(b). This means that the optimal $m_{b}$ for maximum energy does not necessarily maximize time. This can be explained as follows. With larger $m_{b}$, more energy is available. However, the power consumption for hovering increases to reduce the hovering time. Therefore, it is important to carefully choose $m_{b}$ for balanced energy and operation time.

Fig. 3(a) shows the optimal value $m_{b}$ versus $m_{0}$, where $H$ is set to $100 \mathrm{~m}, 300 \mathrm{~m}$ and $500 \mathrm{~m}$. In this figure, (14) is used. One sees that the optimal value $m_{b}$ decreases with increasing $m_{0}$. This is because, when other parameters are fixed, increasing $m_{0}$ results in an overall increase in weight, leading to more energy consumption.

Fig. 3(b) shows the effects of $V_{v}$ on $m_{b}^{*}$. In this figure, (14) is used. One sees that $m_{b}^{*}$ increases with $V_{v}$ first and then approaches an upper limit, which means there should be an optimal $V_{v}$ that exists for fixed $H$.

Fig. 3(c) shows how $m_{b}^{*}$ changes with $V_{h}$. In this figure, $H=$ $300 \mathrm{~m}$ and (14) is used. One sees that $m_{b}^{*}$ increases with $V_{h}$. Taking $D=40 \mathrm{~km}$ as an example, when $V_{h}=15 \mathrm{~km} / \mathrm{s}, m_{b}^{*}$ is about $2 \mathrm{~kg}$. However, according to Fig. 2(b), the available $E_{A 2 G}$ is less than 0 , and this is meaningless. When $V_{h}=30 \mathrm{~km} / \mathrm{s}$, $m_{b}^{*}$ is beyond $m_{b, \max }=20 \mathrm{~kg}$. Thus, $V_{h}$ should be carefully chosen.

\section{CONCLUSION}

In this letter, the optimal weight for a battery in UAV electrical propulsion system has been studied. Numerical results have shown that the optimal battery mass that maximizes the flight performance is determined by $m_{0}$, vertical/horizontal flight speed $V_{v} / V$, flight height $H$ and flight distance $D$. The larger $H$ and $D$ are, the smaller $m_{b}^{*}$ will be. Besides, the optimal battery mass for maximum energy does not necessarily maximize the operation time. Due to the space limitation, several interesting problems remain but will be for future works, such as the joint optimization of transmission power, time and altitude to optimize energy efficiency and the minimization of battery weight for fixed $E_{A 2 G}$. Energy efficiency determines the actual use of the maximized available energy and can be optimized via power and time allocation too.

\section{REFERENCES}

[1] "UAV Engine Manufacturers - Propulsion Systems for UAVs, UGVs, AUVs, USVs - Unmanned Systems Technology." https://www.unmannedsystemstechnology.com/category/supplierdirectory/propulsion-power/uav-engines-propulsion-systems/.

[2] Z. Yang, C. Pan, K. Wang, and M. Shikh-Bahaei, "Energy Efficient Resource Allocation in UAV-Enabled Mobile Edge Computing Networks," IEEE Trans. Wirel. Commun., vol. 18, no. 9, pp. 4576-4589, Sep. 2019.

[3] J. K. Stolaroff, C. Samaras, E. R. O'Neill, A. Lubers, A. S. Mitchell, and D. Ceperley, "Energy use and life cycle greenhouse gas emissions of drones for commercial package delivery," Nat. Commun., vol. 9, no. 1, pp. $1-13,2018$.

[4] M. K. Mohamed, S. Patra, and A. Lanzon, "Designing electric propulsion systems for uavs," in Lecture Notes in Computer Science (including subseries Lecture Notes in Artificial Intelligence and Lecture Notes in Bioinformatics), 2011, vol. 6856 LNAI, pp. 388-389.

[5] T. Chang and H. Yu, "Improving Electric Powered UAVs' Endurance by Incorporating Battery Dumping Concept," in Procedia Engineering, 2015, vol. 99 , pp. $168-179$.

[6] A. Abdilla, A. Richards, and S. Burrow, "Endurance optimisation of battery-powered rotorcraft," in Lecture Notes in Computer Science (including subseries Lecture Notes in Artificial Intelligence and Lecture Notes in Bioinformatics), 2015, vol. 9287, pp. 1-12.

[7] O. Gur and A. Rosen, "Optimizing electric propulsion systems for unmanned aerial vehicles," J. Aircr, vol. 46, no. 4, pp. 1340-1353, 2009.

[8] H. V. Abeywickrama, B. A. Jayawickrama, Y. He, and E. Dutkiewicz, "Empirical Power Consumption Model for UAVs," in IEEE Vehicular Technology Conference, Jul. 2018, vol. 2018-August.

[9] K. A. O. Suzuki, P. Kemper Filho, and J. R. Morrison, "Automatic battery replacement system for UAVs: Analysis and design," J. Intell. Robot. Syst. Theory Appl., vol. 65, no. 1-4, pp. 563-586, Jan. 2012.

[10] H. Yan, Y. Chen, and S.-H. Yang, "Analysis of energy transfer efficiency in UAV-enabled wireless networks," Phys. Commun., vol. 37, p. 100849, Dec. 2019.

[11] H. Yan, Y. Chen, and S.-H. Yang, "UAV-Enabled Wireless Power Transfer with Base Station Charging and UAV Power Consumption," IEEE Trans. Veh. Technol., pp. 1-1, Aug. 2020.

[12] "Li-polymer Battery: Substance or Hype? - Battery University." https://batteryuniversity.com/learn/article/the_li_polymer_battery_ substance_or_hype.

[13] M. Gatti and F. Giulietti, "Preliminary design analysis methodology for electric multirotor," in IFAC Proceedings Volumes (IFAC-PapersOnline), 2013, vol. 2, no. PART 1, pp. 58-63.

[14] S. Park, L. Zhang, and S. Chakraborty, "Design space exploration of drone infrastructure for large-scale delivery services," in IEEE/ACM International Conference on Computer-Aided Design, Digest of Technical Papers, ICCAD, Nov. 2016, vol. 07-10-November-2016.

[15] Y. Zeng, J. Xu, and R. Zhang, "Energy Minimization for Wireless Communication With Rotary-Wing UAV," IEEE Trans. Wirel. Commun., vol. 18, no. 4, pp. 2329-2345, Apr. 2019.

[16] Z. Yang, W. Xu, and M. Shikh-Bahaei, "Energy Efficient UAV Communication with Energy Harvesting," IEEE Trans. Veh. Technol., vol. 69, no. 2, pp. 1913-1927, Feb. 2020.

[17] A. Al-Hourani, S. Kandeepan, and S. Lardner, "Optimal LAP Altitude for Maximum Coverage," IEEE Wirel. Commun. Lett., vol. 3, no. 6, pp. 569-572, Dec. 2014. 